\title{
Strafrecht in der Europaischen Union
}

Citation for published version (APA):

Klip, A. H. (2004). Strafrecht in der Europaischen Union. Universiteit Maastricht. https://doi.org/10.26481/spe.20040429ahk

Document status and date:

Published: 29/04/2004

DOI:

10.26481/spe.20040429ahk

Document Version:

Publisher's PDF, also known as Version of record

\section{Please check the document version of this publication:}

- A submitted manuscript is the version of the article upon submission and before peer-review. There can be important differences between the submitted version and the official published version of record.

People interested in the research are advised to contact the author for the final version of the publication, or visit the DOI to the publisher's website.

- The final author version and the galley proof are versions of the publication after peer review.

- The final published version features the final layout of the paper including the volume, issue and page numbers.

Link to publication

\footnotetext{
General rights rights.

- You may freely distribute the URL identifying the publication in the public portal. please follow below link for the End User Agreement:

www.umlib.nl/taverne-license

Take down policy

If you believe that this document breaches copyright please contact us at:

repository@maastrichtuniversity.nl

providing details and we will investigate your claim.
}

Copyright and moral rights for the publications made accessible in the public portal are retained by the authors and/or other copyright owners and it is a condition of accessing publications that users recognise and abide by the legal requirements associated with these

- Users may download and print one copy of any publication from the public portal for the purpose of private study or research.

- You may not further distribute the material or use it for any profit-making activity or commercial gain

If the publication is distributed under the terms of Article $25 \mathrm{fa}$ of the Dutch Copyright Act, indicated by the "Taverne" license above, 


\title{
STRAFRECHT IN DER EUROPÄISCHEN UNION
}

\author{
ANTRITTSREDE \\ UNIVERSITÄT MAASTRICHT, 29. APRIL 2004
}

Prof. André Klip

Eindversie 2 december 2004

\section{Einleitung}

Am 7. Februar 1992 wurde der Vertrag über die Europäische Union hier in dieser Stadt verabschiedet. Mit diesem Vertrag wurde eine Entwicklung in Gang gesetzt, durch die das Strafrecht nicht länger als einzig nationale Angelegenheit betrachtet werden kann. Bewusst oder unbewusst wurden Schritte eingeleitet, die zu einem einheitlichen europäischen Strafrechtssystem führen könnten. Meine heutige Rede beschäftigt sich hauptsächlich mit der Bedeutung dieser fundamentalen und strukturellen Veränderung von nationaler auf europäische Ebene für die Gesetzgebung, die Strafrechtspolitik und die Strafrechtswissenschaften. ${ }^{1}$

\section{Die Bedeutung des Strafrechts in der derzeitigen Gesellschaft}

Das Strafrecht genießt heut viel Aufmerksamkeit in den Medien, der Regierung und der Politik. In diesem Sinne geht es dem Strafrecht außerordentlich gut. Strafrechtsbezogene Themen fehlen nicht in den neuen Bulletins. Fast jeder Bürger hat seine Meinung darüber, was und wer bestraft werden sollte, wie das geschehen sollte und wie hart die Strafe sein sollte. Ich heiße es willkommen, dass mein Fach eine so zentrale Rolle in der Gesellschaft einnimmt. Doch all diese Aufmerksamkeit hat auch seine Nachteile. Die Erwartungen an das, was mit dem Strafrecht erreicht werden kann, sind sehr hoch - zu hoch meiner Meinung nach. ${ }^{2}$ In vielen Fällen ist das Strafrecht - zumindest nach der öffentlichen Meinung - zum primum remedium geworden: zum ersten Mittel, das gegen ungewolltes Verhalten eingesetzt wird. Diese Entwicklung ist nicht nur niederländisch, sonder hat auch in anderen Ländern und sicherlich auch in der Europäischen Union so stattgefunden. ${ }^{3}$ Und wenn es einen Protagonisten der Idee des Strafrechts als primum remedium gibt, dann ist das die Europäische Union. Dies drückt sich bis heute in einem unablässigen Strom von strafrechtlichen Instrumenten aus.

In den Niederlanden gab es Vorschläge, Eltern für nachlässiges Erziehen zu bestrafen, wenn ihre Kinder Straftaten begangen haben. ${ }^{4}$ Vielleicht wird auch bald weibliche Beschneidung strafbar sein, wenn die Operation im Ausland vorgenommen wurde, und Mädchen aus Risikogruppen sollten regelmäßig daraufhin untersucht werden. Die Verlängerung von verschiedenen Verjährungsfristen fördert die Ausweitung der

\footnotetext{
${ }^{1}$ Das heißt nicht, dass die Europäische Union nicht von anderen Einheiten, zum Beispiel den USA, beeinflusst werden kann. Siehe hierzu Joachim Vogel, Europäische Kriminalpolitik - europäische Strafrechtsdogmatik, Goltdammer's Archiv für Strafrecht 2002, S.517-534.

${ }^{2}$ P.J. van Koppen, Verankering van rechtspraak, Over de wisselwerking tussen burger, politie, justitie en rechter, Antrittsrede Vrije Universiteit Amsterdam 2003, S.65.

${ }^{3}$ Siehe auch J. de Hullu und E.A.M. Verheijen, De beoordeling van Eurostrafrecht, Delikt en Delinkwent 2002, S.696-697.

${ }^{4}$ Brief des Staatssekretärs im Justizministerium, Niet-vrijblijvende vormen van opvoedingsondersteuning, Dokumente der 2. Kammer 1999-2000, 27197, Nr.1, S.11.
} 
Allmacht des Strafrechts. Strafrecht erweitert sich fortwährend: in der Anzahl von Straftaten und involvierten Personen, in Tatorten und Tatzeiten. Als Instrument hat das Strafrecht einen unberührbaren Status mit einer starken moralischen Prägung erreicht. $^{5}$

Kürzlich ist ein neuer Aspekt hinzugekommen, der den Status des Verurteilten verewigt und ihn absolut macht: Es gibt Aufrufe die Nachbarschaft zu informieren, wenn ein Sexualverbrecher unter ihnen lebt; es gibt die Versuche, Niederländern der zweiten Generation die niederländische Staatsangehörigkeit wegzunehmen oder Verurteilte von Einstellungen auszuschließen. Solche Aktivitäten schließen Menschen für immer von der Gesellschaft aus. Die Wissenschaft muss ein Beispiel für die Gesellschaft setzten um ihre Einstellung klar zu machen und um zu erklären, dass Strafrecht nicht ausschließt, sondern einschließt. Das bedeutet, dass Dinge irgendwann vorbei sind, und dass jede bestrafte Person ohne Bedingung wieder in die Gesellschaft zurückkehrt, nachdem sie ihre Zeit abgesessen hat, und dass es dann keinen Platz mehr für neue Sanktionen, Verbannungen oder Anprangerungen gibt. ${ }^{6}$

In diesem Zusammenhang ist es nicht erstaunlich, dass Politiker (europäische wie niederländische) versuchen, immer mehr Kontrolle über die tatsächliche Strafrechtsumsetzung zu gewinnen. Dadurch vermitteln Politiker teilweise das Bild, dass diese Rechtsumsetzung bei denen, die verantwortlich dafür sind, nicht (oder zumindest nicht immer) in guten Händen ist. Nach Anordnung des niederländischen Parlaments (der Tweede Kamer) mussten die außergerichtlichen Übereinkünfte mit gewissen Grossunternehmen der Überprüfung des Justizministeriums unterworfen werden. Mit der Einführung von Mindeststrafen sind Richter gezwungen beträchtliche Strafen anzuordnen. ${ }^{7}$ Politiker, auch Mitglieder des niederländischen Kabinetts, äußern sich oft herablassend darüber, wie mit einzelnen Strafrechtsfällen umgegangen wird. Ihre Kritik richtet sich dabei nicht gegen das Strafrecht an sich, sondern gegen die, die es ausführen: Die Polizei, die keine Diebe stellt; die Staatsanwaltschaft, die nicht, oder nur wegen leichter Straftaten Anklage erhebt; und das Gericht, das zu milde urteilt. ${ }^{8}$

Von ähnlicher Natur sind die unentwegten Klagen auf europäischem Level von der Europäischen Kommission über die Nichtverfolgung von EG-Betrug. Nach Meinung der Kommission ist die Strafverfolgung der nationalen Behörden so schlecht, dass sie es gerne selber übernehmen würde. Die Einrichtung ihrer eigenen Anti-Betrugseinheit war nicht genug für die Kommission, und jahrelang plädierte sie für die Einrichtung eines europäischen Staatsanwaltes. All das sind Versuche, den Nutzen von Strafrecht zu erhöhen. Dies verleumdet die Fähigkeiten des Strafrechts und ignoriert die Tatsache, dass es unmöglich ist, jede Straftat zu verfolgen. Wenn die Kapazität des gesamten Strafrechtsystems die gleiche bleibt, erhöht sich automatisch die Zahl der

\footnotetext{
${ }^{5}$ Buruma wies auf den moralischen Charakter kürzlicher Änderungen im materiellen Strafrecht hin. Y. Buruma, Grenzen aan de strafrechtelijke aansprakelijkheid, in: Glijdende schalen, Liber amicorum J.de Hullu, Wolf 2003, S.76.

${ }^{6}$ Siehe auch C.H. Brants, Over levende gedachten, Antrittsrede Utrecht 1999.

${ }^{7}$ Aber denselben Gerichten werden auch mehr und mehr vage Gesetze vorgelegt. Das verstärkt ihre Urteilsfreiheit.

${ }^{8}$ Siehe für eine interessante empirische Untersuchung zum Vertrauen der Bürger in die Rechtssprechung: T. van der Meer, Vertrouwen in de rechtspraak, rechtstreeks 1/2004, S.9-55 und P.J. van Koppen, der behauptet, (S. 1) dass das Strafrecht als solches kritisiert wird, doch in seinen Untersuchungen sieht man, dass sich die Kritik gegen verschiedenste Funktionäre in der ganzen Kette richtet.
} 
Straftaten, die unbeantwortet bleiben. Wie Boutellier sagt, kann man in dieser Hinsicht von einem permanenten Gefühl der Krise sprechen. ${ }^{9}$ So gesehen geht es dem Strafrecht gar nicht so gut.

\section{Die Aufgabe der Strafrechtswissenschaft}

Das ist eine Feststellung, die die Wissenschaft sich einmal ansehen sollte; sie sollte sich mit der Entwicklung und Gestaltung des Strafrechts beschäftigen. Was ich hier sage ist nichts Neues, und ist nun schon jahrelang so gewesen. Neu ist, dass der Einfluss der Wissenschaft auf Strafrechtspolitik und Gesetzgebung abnimmt. Wie kann dieser erklärt werden? Meiner Meinung nach ist der wichtigste Grund die völlig andere Arbeitsmethode der Medien, die ein sehr wichtiger Faktor in der Wissenschaft sind. Die schnelle schwarz-weiß Berichterstattung, die auf Kontroversen aus ist, passt nicht zu den feinen Nuancen und größeren Zusammenhängen unseres Metiers.

Auf bestimmte Art und Weise sind Politiker immer medienabhängiger geworden. Ein Politiker, der öffentlich sagt, dass die Auslöschung allen Übels unmöglich ist, wird von den Medien zu jemandem gemacht, dem es nichts ausmacht, wenn Straftaten begangen werden. Welcher Politiker hat den Mut zu sagen, dass der Versuch, einen 29 Jahre alten Mordfall zu lösen, auf die Kosten der heute zu untersuchenden und anzuklagenden Verbrechen geht? ${ }^{10}$

Die Medien vermitteln oft ein falsches Bild der Realität der Rechtshandhabung. ${ }^{11}$ Das Bild von Verbrechen und Gerichten, das uns in den Medien gezeigt wird, unterscheidet sich so stark von der Wirklichkeit, dass dazu eine eigene Studien lohnen würde. Ich nenne nur die unbewiesene Vorstellung, dass in den Niederlanden ein mildes Strafklima herrsche. Unsere Bestimmungen bezüglich frühzeitiger Entlassung sind nachweisbar strenger als in den meisten anderen Staaten und unsere Verbrecher leisten einen größeren Teil der ihnen auferlegten Strafe ab. Mein erster Eindruck ist, dass unser Strafklima eher strenger ist als das in anderen europäischen Ländern. Doch um diese Behauptung zu beweisen wäre intensive Forschung nötig. Journalisten behaupten weiterhin, dass Verdächtige aufgrund von „Formfehlern“ entlassen werden, obwohl es das seit den neunziger Jahren kaum noch gibt. Täter werden wegen „guter Führung“ frühzeitig aus dem Gefängnis entlassen - ein Kriterium, das seit über zwanzig Jahren im niederländischen Strafrechtssystem nicht mehr angewendet wird. Im Gegensatz zur Wirklichkeit, dass in den Niederlanden lebenslänglich tatsächlich bis zum Tode vollstreckt wird, liest man immer wieder, dass lebenslänglich Verurteilte in den Niederlanden frühzeitig entlassen werden können.

\footnotetext{
${ }^{9}$ H.Boutellier, De veiligheidsutopie, 2003, 2. Auflage, S.158.

${ }^{10}$ Die Erfahrung mit der Verfolgung von Kriegsverbrechen Jahre nach deren Geschehen lehrt uns, dass große beweisrechtliche Schwierigkeiten durch das Verstreichen der Zeit auftreten können. Im Jugoslawientribunal sind diese Probleme teilweise durch gemeinsame Teilnahmeformen gelöst worden: joint criminal enterprise. Dabei wird die Täterschaft durch eine Art culpa in causa- Schlussfolgerung definiert. Siehe unter anderem: ICTY, Judgement, Prosecutor v. Furundzija, Case No. IT-95-17/1-T, T. Ch.II, 10 December 1998, Klip/ Sluiter III-685, par. 216; ICTY, Judgement, Prosecutor v. Tadic, Case No. IT-94-1-A, A.Ch., 15 July 1999, Klip/ Sluiter III-761, par.220.

${ }^{11}$ Brants, S.34-36.
} 
Tatsachen und Fiktion werden vermischt. Was wirklich mit dem Verbrechen geschieht ist nicht mehr relevant, was zählt, ist der gute Ruf. ${ }^{12}$ So wie Verbrechen dargestellt wird, ist das wichtigste, dass wir es mit Verbrechern zu tun haben. Die Vorstellung, dass im Endeffekt ein europäischer Verbrecher ein europäischer Bürger ist, dass er aus der europäischen Gesellschaft stammt und er daher Freiheit, Sicherheit und Gerechtigkeit beanspruchen kann, fehlt vollständig. De facto ist die Anprangerung aus dem Mittelalter durch die Presse wieder in unsere moderne Gesellschaft zurückgekehrt. Aus dieser Sicht ist es gerechtfertigt, dass Richter bei ihren Entscheidungen mit in Betracht ziehen, in welchem Maße Verdächtige den Medien ausgesetzt waren. Dadurch werden die Medien ein Teil der Vollstreckung der Strafe und damit Teil der Ausführung der Wirklichkeit. Ich fürchte, dass eine solche Entwicklung, bei der Strafpolitik auch von Mediengeschehnissen mitbestimmt wird, durch Europa gestärkt wird. Dabei kann Fiktion zu Wirklichkeit werden. Diese klaffende Lücke kann zu einem Wettkampf werden, bei dem zu viel beansprucht wird, gefolgt von neuen Kompetenzen, gefolgt von einem weiteren Rückgang des tatsächlichen Einflusses des Strafrechts auf die Gesellschaft. Hierin liegt eine Gefahr für die Rechtssicherheit und den Rechtsschutz. Die Ausdehnung der Kompetenzen verstärkt die Position des Staates, aber auch seinen Handlungsspielraum und erweitert die Wahrscheinlichkeit von Willkür. Auf dieses Phänomen hinzuweisen ist die Pflicht der Wissenschaft, damit ein Teufelskreislauf durchbrochen werden kann. Das kann dadurch erreicht werden, dass das Strafrecht selbst als Durchsetzungsinstrument in Frage gestellt wird.

Wenn Verbrechen als lösbares Problem dargestellt wird, besteht das Risiko, das solche Mittel benutzt werden, die zu einem solchen unerreichbaren Ziel passen. Meines Erachtens sollte das Strafrecht viel von seiner Unverwundbarkeit einbüßen und auf eine weit bescheidenere Stelle auf der Skala gesetzt werden. Das soll jedoch nicht dazu führen, dass die in der Gesellschaft geäußerten Klagen über Sicherheitsmangel mit dem Verweis auf „gesundes Volksempfinden“ herunter gemacht werden. Zwischen faschistischem Missbrauch der Justiz und dem Mangel an Sinn für die Bedürfnisse der Bevölkerung ist einiger Raum. Schließlich existiert das Strafrechtssystem für die Bevölkerung, und es operiert nicht in einer sterilen, abstrakten und theoretischen Situation eines Laboratoriums. Das Strafrecht soll der Gesellschaft dienen, doch genau deswegen sollte es greifbar und vertrauenswürdig sein. ${ }^{13}$ Unsere soziale Aufgabe besteht darin, die Gesellschaft darüber zu informieren, was getan werden kann und was nicht. Wir müssen immer wieder auf bestimmte Tatsachen und Konsequenzen hinweisen und die richtigen Fragen stellen, auch wenn die Erklärung etwas länger dauert. Wenn Politiker nach fertigen Lösungen fragen, die es nicht gibt, muss ihnen geantwortet werden, dass dies nicht möglich ist. ${ }^{14}$ Ich mache mir keine Sorgen um eine Entfremdung zwischen Politikern und Akademikern; es ist immer so gewesen und es festigt den notwendigen kritischen Abstand. ${ }^{15}$

Auch auf einer anderen Ebene kann das Strafrecht diskutiert werden. In einer Zeit, in der neue Normen und moralische Werte festgelegt werden, kann dies eventuell zu einer neuen Definition von grundlegenden Strafrechtsprinzipien führen. Das mag anfangs Angst einflößend klingen, aber für mich klingt es wünschenswert, da wir in

\footnotetext{
${ }^{12}$ G.J.M. Corstens, Een stille revolutie, Arnhem 1995, S.14.

13 J. de Hullu, Recidive en straftoemeting, Deventer 2003, S.4.

${ }^{14} \mathrm{~J}$. de Hullu, Recidive en straftoemeting, Deventer 2003, S.11.

${ }^{15}$ Siehe auch Dokumente der 2. Kammer, 1991-1992, 22268, Nr.5, S.5.
} 
den Niederlanden aus dogmatischer Sicht in die Irre gelaufen sind. Unterscheidungen zwischen Täterschaft, Teilnahme und Beihilfe an einer Tat, die früher notwendig waren, sind in der Rechtssprechung verkommen zu einem einfachen: Du warst dabei und dafür bist $\mathrm{Du}$ dran! Und ich habe noch nicht einmal mit der Weite der Komplizenschaft in einer kriminellen Organisation angefangen, auf die in Artikel 140 des niederländischen Strafgesetzbuches Bezug genommen wird. Diese Entwicklung steht den grundlegenden Prinzipien des klassischen Strafrechts entgegen, das als sein actus reus (schuldige Handlung) ein Vorsatz verlangt (der Täter muss die tat gewollt haben) und nur auf Kontrollinstrumente zurückgreift, wenn andere Methoden versagen. In der Praxis wurde auch das Schuldprinzip in vielen Bereichen aufgegeben und strikte Haftung im Strafrecht eingeführt. ${ }^{16}$

Heißt das, dass das aktuelle Strafrecht nicht mehr auf dem neusten Stand ist? Müssen wir neue Lehren suchen? Und wenn, sollten wir das dann nicht lieber in der Union tun als auf nationalem Level? Viele technische Entwicklungen sind aufgetaucht, die am Ende des 19. Jahrhunderts, als unser Strafgesetzbuch geschrieben wurde, noch nicht existierten. Der Platz der Niederlande in der Welt hat sich verändert. Das war auch das Ergebnis des Justizministeriums, welches zum Ausdruck brachte, dass wir ein System finden sollten, das besser in unsere Zeit passt. ${ }^{17}$ Das klingt richtig für mich. Aber ich vermisse noch immer die fundamentale Diskussion über neue Grundprinzipien. Mir persönlich geht es dabei mehr um die Frage, welche Rolle wir dem Strafrecht in unserer Gesellschaft einräumen wollen und wie Täterschaft und Teilnahme definiert werden, als um detaillierte Fragen darüber, ob die Bestrafung von integrierten Ausländern in den Niederlanden als Sextouristen möglich ist, wie es nun im Artikel 5a des Strafgesetzbuches geregelt ist. In einer abstrakten Wissenschaft wie der Rechtswissenschaft bleibt nichts für immer bestehen, alles ist dem ständigen Wandel unterworfen. Das kann zu einem Überdenken von Konzepten führen, die lange Zeit ein Existenzrecht hatten, aber es kann auch zu der Einsicht führen, dass einige Rechte nicht abgeschwächt werden können, ohne dass dabei Prinzipien eines demokratischen Rechtsstaates aufgegeben werden.

\section{Welche Rolle hat die Europäische Union im Strafrecht?}

Es wird Zeit, dass ich Ihnen erkläre, was ich mit Strafrecht in der Europäischen Union meine - schließlich sind Sie gekommen, um mich darüber sprechen zu hören. Ich möchte Strafrecht in der Europäischen Union definieren als die Gesamtheit der Normen der Europäischen Gemeinschaft und der Europäischen Union, die eine strafrechtliche Dimension haben. Diese können in drei Ebenen aufgeteilt werden:

1) Die nationale Ebene:

- die strafrechtliche Umsetzung des Gemeinschaftsrechtes (freiwillige Wahl des Einsatzes von Strafrecht/ Handhabung des Gemeinschaftsrechts ist verpflichtend); ${ }^{18}$ sowie

\footnotetext{
${ }^{16}$ Siehe D. Roef, Strafrechtelijke verantwoordelijkheid in de risicomaatschappij, in: P.L. Bal, E. Prakken, G.E. Smaers, Veiligheid of vergelding?, Deventer 2001, S.33-36.

${ }^{17}$ Strafrecht in einer veränderten Umgebung (Strafrecht in een veranderende omgeving), Rede des Justizministers Donner am 1. December 2003 an der Katholischen Universität Nijmegen.

${ }^{18}$ Siehe J.A.E. Vervaele, Handen en tanden van het gemeenschapsrecht, Antrittsrede Utrecht 1994.
} 
- die Umsetzung des von der Union als strafbar festgestellten Verhaltens. (wie es in Gemeinsamen Maßnahmen des Rats, Rahmenbeschlüsse und Konventionen enthalten ist);

2) die zwischenstaatliche Ebene:

- die Regulierung administrativer und strafrechtlicher Zusammenarbeit (die de facto zu einer gemeinsamen Handhabung führt);

3) die supranationale Ebene:

- das echte (supranationale) europäische Strafrechtssystem mit direkter Handhabung auf europäischer Ebene. Mit der Ausnahme der Handhabung im Bereich des Wettbewerbsrechtes ist dies nirgends anders verwirklicht worden, es gibt aber Vorschläge. ${ }^{19}$

Im Folgenden will ich in meiner Rede besonders über den Zusammenhang zwischen der nationalen und der supranationalen Ebene sprechen, ebenso wie über die Gestaltung eines europäischen Strafrechtssystems.

Vor 35 Jahren, am Vorabend des Abschlusses des Vertragsnetzwerkes über internationale Rechtshilfe des Europarates, das den Staaten die Möglichkeit gibt, sich gegenseitig in jeder Phase der Verbrechensaufklärung, des Prozesses und der Vollstreckung zu helfen, sagte Van Agt in seiner Antrittsrede Über ein extrovertiertes Strafrecht: „Die Strafe von nationalen Behörden ist eigentlich nicht auf den Schutz einzelner nationaler Gemeinschaften gerichtet, sondern auf das Wohlergehen der Menschen in ihrer grenzüberschreitenden Gesellschaft. In dieser Hinsicht ist es angemessen, den nationalen Richter auch mit der Umsetzung solcher Normen zu betrauen, die anderswo aufgestellt wurden, solange eine gerechtere und zweckmäßigere (internationale) Arbeitsteilung bei der Strafverfolgung erreicht wird.“20 Zehn Jahre später, im Jahre 1979, torpedierte selbiger Van Agt - nun Premierminister der Niederlande - mit einem niederländischen Veto den Plan des französischen Präsidenten Giscard d' Estaing zu einem espace judiciaire. ${ }^{21}$ Seit dem hat sich vieles verändert.

Erstens ist er Einfluss des Vertragsrechtes auf das Strafrecht sehr viel stärker geworden. Ich habe einmal ausgerechnet, wie lange die Niederlande im letzten Jahrhundert gebraucht haben, fünfzig Verträge mit strafrechtlicher Relevanz abzuschließen. Vom 18. April 1904 bis zum 28. August 1954 (50 Jahre), dann bis zum 8. August 1975 (21 Jahre), zum 20. Dezember 1988 (13 Jahre), von da bis zum 10. März 1995 (fast sieben Jahre), dann bis zum 21. Mai 1997 (zwei Jahre), zum 25. Mai 2000 (drei Jahre) und dann bis zum September 2003 (drei Jahre und vier Monate). Auf den ersten Blick scheint es als ginge die internationale Produktion von Verträgen zurück. Doch das ist nicht der Fall. Nur werden heute immer mehr europäische Rechtsinstrumente in anderen Formen erlassen. Ich habe mit der

\footnotetext{
${ }^{19}$ Auf Grund der Interpretation des EuGHMR des Art. 6 EMRK verstehe ich das EGWettbewerbsrecht als Strafrecht. Ich verfolge dabei also eine andere Idee als Corstens, der das EUStrafrecht in drei Teile einteilt: 1 . die strafrechtliche Zusammenarbeit; 2. Menschenrechte, basierend auf der EMRK; 3. Die Ausarbeitung von Gemeinschaftrecht ins nationale Recht, siehe G.J.M. Corstens, Europees strafrecht, in: Rede en Recht, Liber amicorum Nico Keijzer, Deventer 2000, S.1125.

${ }^{20}$ A.A.M. van Agt, Naar een extravert strafrecht, Antrittsrede, Katholische Universität Nijmegen 1969.

${ }^{21}$ Siehe Anhang zu Dokumenten der 2. Kammer des Parlaments, 1978-1979, Nr.1261.Siehe auch A. Weyemberg, L’harmonisation des législations: condition de l'espace pénal européen et révélateur de ses tensions, Bruxelles 2004, S.14-15.
} 
Fleißarbeit begonnen, all diese gemeinsamen Akte, Standpunkte, Empfehlungen, Entscheidungen und Rahmenbeschlüsse herauszusuchen, aber da habe ich noch viel zu tun. Nicht nur die Europäische Union, sondern auch andere Organisationen wie die Vereinten Nationen haben zu einer stufenweisen Verschiebung des Vorrangs der Gesetzgebung im Strafrecht von einer nationalen auf eine supranationale Ebene beigetragen.

Ich schätze, dass diese Entwicklung sich verintensivieren wird, besonders in Europa. Die Erklärung für diese Entwicklung ist in der vorher genannten sozialen Rolle des Strafrechts und dem hohen Sockel zu suchen, auf den die Mitgliedsstaaten es gesetzt haben. Der Vertrag zur Europäischen Union schreibt in seinem Artikel 29 vor, dass die Union das Ziel verfolgt, „den Bürger in einem Raum der Freiheit, der Sicherheit und des Rechts ein hohes Maß an Sicherheit zu bieten“. Diese Ambition kann als Kodifikation der Sicherheitsutopie charakterisiert werden, die von Boutellier so treffend beschrieben wurde. ${ }^{22}$ Es verbindet ein gesellschaftliches Gefühl mit den rechtlichen Möglichkeiten und will dadurch die Position des Strafrechtes in der europäischen Gesellschaft und den Zusammenhang zwischen nationalem Strafrecht und jenem der EU bestimmen. Der Vertag verstärkt zusätzlich den verbrauchergemäßen Anspruch der Bürger auf Sicherheit gegenüber der (europäischen) Regierung direkt. ${ }^{23}$ Anders als zu Zeiten eines Van Agt oder Giscard d'Estaing (in seinem ersten politischen Leben) steht das Strafrecht heute unter Druck, da es kein effektives Mittel zur Ausrottung der Kriminalität zu sein scheint. Souveränität wird in diesem Fall als Behinderung der Effektivität angesehen. ${ }^{24}$ Eher muss pessimistischerweise erwartet werden, dass die Politiker dem Druck nicht werden widerstehen können, immer mehr strafrechtliche Kompetenzen an die Europäische Union abzugeben. Anschließend wird dies den Druck auf die Union erhöhen, mehr Umsetzung selbst in die Hand zu nehmen. Aus dem Vertrag von Maastricht entsteht also ein verstärktes gesellschaftliches Interesse am Strafrecht, an einem europäischen Zuschnitt dafür. ${ }^{25}$

Ist das Strafrecht bei der Europäischen Union in den richtigen Händen? Die Antwort auf diese Frage ist im Moment negativ. Immerhin gibt es viele Beispiele von Gesetzgebung ohne erkennbare Notwendigkeit oder ohne Notwendigkeit europäischen Eingreifens. 1997, kurz nach der Festnahme von Dutroux, wurde eine Gemeinsame Maßnahme verkündet, die unter anderem auf den Kampf gegen internationale Netzwerke der Kindermisshandlung gerichtet war. Im aktuellen Strafprozess ist von der grenzüberschreitenden Komponente nichts übrig geblieben. ${ }^{26}$ Irgendwann als Entwurf nach den Attentaten des 11. September begonnen, führte die

\footnotetext{
${ }^{22}$ Siehe weiterhin H. Boutellier, De veiligheidsutopie, 2 Auflage 2003, S.47. Dies taucht mit gleichem Wortlaut in den Artikeln 41 und III-158 des Europäischen Verfassungsentwurfs wieder auf.

${ }^{23}$ Das wirft die Frage auf, ob es für die Bürger noch eine eigene Rolle (Pflicht) gibt, Frieden, Sicherheit und Gerechtigkeit herzustellen. Auch hier eine Verschiebung: Der Vertrag von Amsterdam wollte Europa näher an den Bürgern bringen, der Verfassungsentwurf will nun die Bürger näher an Europa bringen.

${ }^{24}$ Siehe u. a. C. Rijken, Trafficking in Persons, diss. Universiteit van Tilburg 2003, S.9.

${ }^{25}$ Die Europäischen Freiheiten nähren paradoxerweise die Unsicherheit. Siehe Ralf Dahrendorf, De Volkskrant 16. April 2004, S.13.

${ }^{26}$ Siehe A. Weyembergh, S. 344: "les grandes difficultés auxquelles sont confrontés les travaux de rapprochement des législations pénales accroissent la tentation de suivre l'événementiel et d'exploiter les drames de l'actualité pour obtenir le consensus nécessaire, ce qui favorise le recours à des définitions larges négociées et adoptées dans l’urgence, sans réflexion approfondie.”
} 
EU einen europäischen Haftbefehl ein, denn es wurde unter anderem argumentiert, dass zu viele Auslieferungen abgelehnt würden und dass die doppelte Strafbarkeit der Zusammenarbeit im Wege stünde. Daten über eine große Anzahl von Ablehnungen sind jedoch nie gemacht worden. Es gibt also Gesetze für Probleme, die gar nicht existieren. ${ }^{27}$ Die rechtsvergleichenden Studien, die von der Union teilweise zur Unterstützung eines Gesetzesentwurfes oder zur Kontrolle der Durchsetzung betrieben werden, erfüllen nicht die Vorraussetzungen, die an sie gestellt werden. Sie bleiben in normativen Vergleichen stecken, ohne den Kontext oder die tatsächliche Durchführung mit einzubeziehen.28 Das Phänomen der Anzeigetafel (scoreboard), welches Mitgliedsstaaten zum Bericht über die Ratifizierung und Ausführung einzelner Gesetze und über die zuständige Behörde verpflichtet, lässt Erinnerungen an den Fünf-Jahres-Plan der Sowjetunion wieder wach werden. Auch die wurden formell immer erfüllt, verzogen aber die Wirklichkeit. Die wachsende Wichtigkeit der Europäischen Union sollte meines Erachtens mit einem realistischen Einblick ins europäische Verbrechen einhergehen. Fijnaut rief zuerst zu einer europäischen Forschung auf. $^{29}$ Wenn er damit die Einführung von mehr rechtsvergleichende Studien und europäischer Kriminalitätsforschung zur Vorbereitung und Unterstützung einer Strafrechtspolitik meint, so halte ich das für eine gute Wahl. ${ }^{30}$ In diesem Zusammenhang ist das Fehlen eines europäischen Forschungs- und Dokumentationszentrum ein schwerer Mangel. ${ }^{31}$

Die Union reguliert zwar die Handhabung, aber ausschließlich in ihrer repressiven Funktion und nicht im machtkritischen Sinn. Dies führt zu einem einseitigen Wachstum, auf Kosten von sowohl der Rechtshandhabung als auch des Rechtsschutzes. Meiner Meinung nach ist das ein großes Problem: Nicht die Herkunft der Gesetze, sondern Qualität und Quantität der europäischen Strafgesetze sind eine ständige Sorgenquelle. Die Gesetzgebungsmaschine der Union macht das Strafrecht der EU zu einem immer komplexeren und undurchschaubareren Rechtsgebiet. Mit einer legislativen Pause wäre der europäische Rechtstaat gut beraten. Ich habe bereits häufiger kritisiert, übrigens mit wenig Erfolg, dass es keine Reflexion gibt, und dass nicht einmal abgewartet wird, ob ein bestimmtes Instrument Erfolg hat. ${ }^{32}$ Ein aktuelles Beispiel dieses Schemas ist der Vorschlag für einen Rahmenbeschluss des Rates über die Europäische Beweisanordnung zur Erlangung von Sachen, Schriftstücken, und Daten zur Verwendung in Strafverfahren . In diesem Vorschlag werden EU-Verträge von 2000 und 2001 schon wieder für ersetzt erklärt - ohne dass

\footnotetext{
${ }^{27}$ A.H.J. Swart, Een ware Europese rechtsruimte, Antrittsrede Amsterdam 2001, S.29. Swart wirft hier die Frage auf, ob es nicht einen Zusammenhang gibt mit dem Willen der Staaten, wirklich erfolgreich miteinander zu kooperieren.

${ }^{28} \mathrm{Zu}$ Untersuchungsmethoden und vergleichendem Strafrecht: C.J.C.F. Fijnaut, Rechtsvergelijking en strafrecht(swetenschap): enkele methodologische beschouwingen, Preadvies voor de Nederlandse vereniging van rechtsvergelijking, no.61, Kluwer 2001.

${ }^{29}$ Siehe Fijnaut, preadvies, S.88.

${ }^{30}$ C C. Fijnaut, De Europese Unie: een lusthof voor (strafrechtelijke) rechtsvergelijking, Deventer 2001; idem De uitbreiding van de Europese Unie en de strafrechtsvergelijking, in: De meerwaarde van de rechtsvergelijking: Opstellen aangeboden aan prof. mr. H.U. Jessurun d’Oliveira, S.201-213.

${ }^{31}$ Obwohl es Europäische Zentren für die Beobachtung von Drogen und Drogenabhängigkeit gibt, ebenso wie eines für Rassismus und Fremdenfeindlichkeit.

${ }^{32}$ A.H. Klip, Amsterdams uniestrafrecht, NJB 1998, S.811-816; Neuere Entwicklungen im europäischen Strafrecht und in der europäischen Zusammenarbeit in Strafsachen, in: 22.

Strafverteidigertag vom 20.-22. März in Erfurt, Schriftenreihe der Strafverteidigervereinigungen, Köln 1999, S.39-54. Siehe auch C.J.C.F. Fijnaut, De strafrechtelijke bescherming van de financiële belangen van de Gemeenschap tegen fraude, Delikt en Delinkwent 2000, S.972-988.
} 
sie überhaupt erst in Kraft getreten wären. Auch die bereits erwähnte DutrouxEntscheidung wurde durch einen neuen Rahmenbeschluss ersetzt. ${ }^{33}$

Die Handlungen der Europäischen Union auf der strafrechtlichen Ebene zeigen einen ziemlich problemgesteuerten Charakter. Es ist jedoch schwierig, Zusammenhänge oder ein System zu erkennen. Rasches, symbolisches Handeln wird als wichtiger angesehen als gut durchdachtes Handeln. Meiner Meinung nach arbeitet das europäische Strafrechtssystem kontraproduktiv und die Rechtshandhabung und Rechtsgarantien der Bürger werden geopfert. Ein deutscher Kollege bezeichnete das als Systemschutz-Strafrecht: "Am Horizont taucht also ein Europaweites symbolisches Strafrecht und eine symbolische Strafjustiz auf, alles als Ausdruck des Unvermögens einer Politik, die für strukturelle gesellschaftliche Reformen keine Kraft mehr hat." ${ }^{34}$ Was er damit mein ist, dass das Ausnutzen des Strafrechtes für politische Zwecke zwar zu einer Kontinuität in der Politik führt, nicht aber zu irgendeinem sinnvollen Gebrauch des Strafrechts. Dazu möchte ich gerne hinzufügen, dass dies das Risiko beinhaltet, dass papierne rechtliche Anweisungen in der Praxis unanwendbar sind, und dass die Handhabung dann eher willkürlich von statten geht. Das kann nur dem Vertrauen in die schützende Funktion des Rechts schaden.

\section{Wie weiter? Was könnte zum Strafrecht der Europäischen Union dazugehören?}

Bei der Gelegenheit der Eröffnung von Eurojust, machte der niederländische Justizminister heute vor genau einem Jahr den Vorschlag, eine europäische Sonderautorität für bestimmte grenzüberschreitende Verbrechen zu schaffen, und den Rest der Straftaten den nationalen Behörden zu überlassen. ${ }^{35}$ Kurz gefasst ist es sein Standpunkt, dass es keinen Sinn macht, das gesamte Strafrechtssystem zu harmonisieren, da 90\% aller Verbrechen einzig und allein nationalen Bezug haben. Der Vorschlag des Ministers ist noch nicht genauer ausgearbeitet, aber er ist sehr interessant. ${ }^{36}$ Gerade im Licht der Diskussion um die europäische Verfassung und der kommenden niederländischen Ratspräsidentschaft, können neue Initiativen erwartet werden.

Eine Trennung zwischen europäischem und nationalem Strafrecht würde - wie ich es sehe - ein komplettes europäisches Strafjustizsystem, einen Europäischen Strafgerichtshof, einen europäischen Staatsanwalt und eine europäische Polizei beinhalten, alles auf der Basis von europäischen Gesetzen und unterstützt von einem frei gewählten europäischen Parlaments. Wenn wir das wollen, benötigen wir eine Definition vom europäischen und nationalen Strafrecht. ${ }^{37}$ Jedenfalls baut das Memorandum der Justizminister über die doppelte Strafbarkeit vom März 2004 nicht

\footnotetext{
${ }^{33}$ Rahmenbeschluß des Rates vom 22. Dezember 2003 zur Bekämpfung von sexuellem Missbrauch von Kindern und Kinderpornographie, PB 2004, L 13/44.

${ }^{34}$ P.-A. Albrecht, Europäischer Strafrechtsraum: Ein Albtraum?, Zeitschrift für Rechtspolitik 2004, S.1-4.

${ }^{35}$ Einige Tage später wurde dieser Standpunkt wiederholt in dem Memorandum für einen europäischen Rechtsraum (Een Europese strafrechtelijke ruimte van 9 mei 2003 van de Staatssecretaris voor Europese Zaken, kenmerk DIE-258/03).

${ }^{36}$ Siehe auch Ch.J. Enschedé, Een Uniform Europees Strafrecht? Report für den Europarat 1971, nachgedruckt in Arnhem 1990.

${ }^{37}$ Ich stimme mit der Meinung der Regierung überein, dass eine klare Abgrenzung nötig ist,

Dokumente der 2. Kammer des Parlaments, 2003-2004, 28473, Nr.35, S.4.
} 
auf dem Distanzierungsgedanken des letzten Jahres auf. ${ }^{38}$ Die Regierung hält hierin an der Bedingung der doppelten Strafbarkeit in der Zusammenarbeit mit anderen Mitgliedsländern fest, wenn die Tathandlungen in den Niederlanden begangen wurden. Das soll heißen, dass wenn die Tat in den Niederlanden begangen ist und sie hier nicht strafbar ist, dass dann die Niederlande auch keine Rechtshilfe leisten kann.

Ich möchte ein anderes Model vorschlagen, dass folgendermaßen aussehen könnte: Wenn das Problem darin liegt, dass andere Staaten keine Zuständigkeit für Verbrechen verlangen können, die hier nicht geahndet werden, obwohl sie hier begangen wurden, dann sollte die beschränkte Strafgewalt das leitende Prinzip sein. Das führt zu einer völlig anderen Regel: Strafgewalt innerhalb der Europäischen Union kann dann nur im staatseigenen Gebiet errichtet werden. ${ }^{39}$ Demnach kann Deutschland nicht mehr den Missbrauch von (weichen) Drogen eines deutschen Bürgers in den Niederlanden verfolgen. Aber das bedeutet auch, dass, wenn die Griechen es für notwendig erachten, das Aufschreiben von Flugzeugnummern strafrechtlich zu verfolgen, wir ihnen mit den Nachforschungen und der Verfolgung helfen werden. ${ }^{40}$ Das setzt eine Menge Vertrauen voraus, aber es birgt auch riesige Vorteile für die Rechtsdurchsetzung und für die Rechte der Einzelnen. Es ist immer klar, welcher Staat für die Handhabung verantwortlich ist. Im Grunde führt die heutige Situation der sich überschneidenden Zuständigkeiten zu der ständigen Diskussion zwischen Mitgliedsstaaten, ob ein Verbrechen kriminalisiert werden soll und welches Strafrecht angewendet werden soll. ${ }^{41}$ Die Erfordernisse der doppelten Strafbarkeit abzuschaffen und die strafrechtliche Zuständigkeit auf das Staatsgebiet zu beschränken, auf dem die Tat begangen worden ist, würde außerdem auf dem bestehenden Netzwerk internationaler Zusammenarbeit in Strafsachen aufbauen. ${ }^{42}$ Das Netzwerk der Zusammenarbeit des Europarates hat es bisher nie geschafft, dieses eine wichtige Thema mit einzubeziehen: eine Vorkehrung für Zuständigkeitskonflikte. ${ }^{43}$ Die Erweiterung der Zuständigkeiten über das eigene Staatsgebiet hinaus, wurzelte einst in der Sorge, dass ein Verdächtiger ungestraft davon kommen könnte und dass es darum nötig sein müsste, ihn im eigenen Land zur Verantwortung zu ziehen, ohne die gesamte Strafrechtspolitik eines anderen Landes zu verändern. Die meisten Straftatbestände sind mittlerweile in der einen oder anderen Form in der gesamten Union strafbar. Die verbleibenden Unterschiede sind vielleicht jedoch zu entscheidend, als das man sie vernachlässigen könnte.

\footnotetext{
${ }^{38}$ Memorandum zur Bedingung der doppelten Strafbarkeit im niederländischen Strafrecht. Dokumente der 2. Kammer des Parlaments, 2003-2004, 29451, Nr.1; Vorschlag für ein Rahmenbeschluss des Rates über die Europäische Beweisanordnung zur Erlangung von Sachen, Schriftstücken, und Daten zur Verwendung in Strafverfahren , 25. November 2003, 15221/03.

${ }^{39}$ Ob für extraterritoriale Strafverfolgung eine Vergleichbare Notwendigkeit besteht, ist eine andere Frage.

${ }^{40}$ Ein derartiger Ansatz passt auch besser zum Legalitätsprinzip.

${ }^{41}$ Dann können die Niederlande auch Abstand nehmen von der defensiven und zurückhaltenden Einstellung gegenüber Initiativen zum europäischen Strafrecht; siehe z. B. das Regierungsmemorandum zum Strafrecht und Strafprozessrecht in Europa, Dokumente der 2. Kammer des Parlaments, 1998-1999, 26656, Nr.1, siehe auch P.H.P.H.M.L. van Kempen, Waarborgen tegen de onwrichtende werking van Eurostrafrecht, Glijdende schalen, de Hullubundel, S.247-266.

${ }^{42}$ A.H.J. Swart, Goede rechtsbedeling en internationale rechtshulp in strafzaken, Antrittsrede Utrecht, Deventer 1983.

${ }^{43}$ Verschiedene Versuche, das Problem mit Hilfe der Priorität der verschiedenen Prinzipien zu lösen, missglückten, aber sie führten zu einer interessanten Studie: Council of Europe, Extraterritorial Jurisdiction. Damit würde die EU endlich Zuständigkeitskonflikte verhindern, wie sie sich in Artikel 31 Unterart. c des Unionsvertrages verpflichtet hat.
} 
Die Abgrenzung die ich gerade gezogen habe, betrifft die Beziehung zwischen den Mitgliedsstaaten. Es bringt keine Lösung für schwierige Fälle, in denen der Tatort nicht einfach in einem einzelnen Mitgliedsstatt verortet werden kann. ${ }^{44}$ Diese Fälle müssen auf Unionsebene geklärt werden. Darum müssen wir Kriterien für solche Fälle schaffen, die auf europäischer Ebene behandelt werden.

In dem Memorandum zum „europäischen Strafrechtsraum“, das im letzten Jahr von der Regierung herausgegeben wurde, ist Folgendes zu lesen: „Ein effektiver Kampf gegenüber Verbrechen gegen die Interessen der Union (Fälschung des Euro oder Betrug gegen die finanziellen Interessen der Gemeinschaft) und gegen schweres staatenübergreifendes Verbrechen, was definiert werden muss, sich aber zwischen dem Schmuggeln (il)legaler Ware oder Menschen bis zum Verschmutzen der Umwelt abspielen kann, bedarf im Endeffekt seiner eigenen Jurisdiktion, die von nationalen Grenzen nicht durchschnitten werden kann.”45

Der Justizminister appelliert an kriminologische Erkenntnisse im Hinblick auf die Erscheinungsformen der Kriminalität und besonders auf die Frage, ob die betroffenen Taten tatsächlich grenzübergreifend sind. ${ }^{46}$ Das ist meiner Meinung nach der richtige Weg. Ich empfinde die aktuelle Methodologie als nicht besonders gut durchdacht, erst eine Organisation zu gründen: Europol, die europäische Staatsanwaltschaft, oder neue Straftatbestände einführen, zu Problemen, die kurz vor einem EU-Gipfel in den Medien sind. Am Ende beeinflusst all dies nur das Meinungsbild, nicht aber das Recht selbst.

Die Abgrenzung müsste im materiellen Strafrecht gefunden werden, nicht in den Kooperationsbedingungen. Aus diesem Grunde sollten Straftaten, die Teil eines europäischen Strafrechts sein sollen, bestimmte Bedingungen erfüllen. Was sind diese Bedingungen? Meines Erachtens gibt es drei kumulative:

1. die Tat hat einen grenzüberschreitenden Charakter

2. die Prävention, das Aufklären und Verhandeln eines solchen Falles bringt auf nationaler Ebene mehr Schwierigkeiten mit sich als auf europäischer Ebene

3. die Tat berührt ein europäisches Politikfeld. ${ }^{47}$

\footnotetext{
${ }^{44}$ Abgesehen von dem Problem das es mehr als ein Auslegungsprinzip gibt, siehe H.D. Wolswijk, Locus delicti en rechtsmacht, diss. Utrecht 1998.

${ }^{45}$ Memorandum vom 9. Mai 2003.

${ }^{46}$ Siehe D. van Eck, Het universele karakter van het strafrecht, opstellen over recht en rechtsgeschiedenis, aangeboden aan D. Hermesdorf, Kluwer 1965, S.149-150: “De ontwikkeling naar een internationaal strafrecht, in zoverre dit hetzij bij de inhoudgeving der normen, hetzij bij de handhaving der normen gedeeltelijk de taak van het nationale gezag gaat overnemen, zal een ontwikkeling moeten zijn, die beslist wordt door het criterium, welk organisatorisch verband der samenleving het strafrecht op de meest verantwoorde wijze zal handhaven. Daarbij zal echter rekening mee moeten worden gehouden, dat de uitvoering van het strafrecht slechts één van de vele taken is, die aan het samenlevingsverband der mensen zijn opgedragen. De beslissing omtrent de verdeling van strafrechtelijke competenties mag dus nooit geschieden naar criteria, die louter en alleen betrekking hebben op de uitoefening van het strafrecht. Het belang van een goede handhaving van het strafrecht zal moeten worden gezien in het gehele verband van de taken, waarvoor de menselijke samenleving zich ziet gesteld.”

${ }^{47}$ Siehe meine Conditions for a European Corpus Juris Criminalis, in Michael Faure, Jan Smits and Hildegard Schneider (Hrgb.) Towards a European Ius Commune in Legal Education and Research,
} 
Die betroffenen Straftaten haben einen locus delicti in verschiedenen Ländern, was es schwierig macht, einen einzelnen Staat als den zuständigen zu bestimmen. Man denke nur ans Abgaberecht, an Subventionsbetrug, Geldwäsche, Frauen- und Menschenhandel oder Drogen- und Waffenhandel. Die zweite Bedingung hat etwas mit Effektivität zu tun. Wenn ein Fall grenzübergreifende Aspekte hat, die nationalen Behörden aber ohne Probleme damit umgehen können, so ist kein europäischer Eingriff nötig. ${ }^{48}$ Das dritte Element stellt sicher, dass die verschiedenen Umsetzungsmodalitäten als kohärent angesehen werden, aber es unterstreicht auch den Gedanken der Subsidiarität.

Natürlich wird es Verbrecher geben, die nicht über solch eine Einteilung der gerichtlichen Zuständigkeiten nachdenken, wenn sie ein Verbrechen planen. Tatsachenkomplikationen werden auftreten, in denen Verdächtige europäische und nationale Verbrechen begehen. Auch das Prinzip einer fairen Justizverwaltung, kann bei der Suche nach dem angemessenen Gericht eine entscheidende Rolle spielen. ${ }^{49}$

Der europäische Rechtsbereich führt also zu drei ziemlich gut zu trennenden Zuständigkeitsebenen: der nationalen, der europäischen und der außer-europäischen. ${ }^{50}$ Ich bin weniger begeistert über die Pläne der Europäischen Kommission, einen europäischen Staatsanwalt zu schaffen, wie es im Corpus Juris beschrieben ist. Sie führen zu einem hybriden System, welches zwischen nationaler und europäischer Umsetzung steht. ${ }^{51}$ Die Vorschläge für die Artikel III-171ff im europäischen Verfassungsentwurf bekräftigen diesen hybriden Charakter und bieten keinen neuen Ansatz. Ähnliche Einwände bestehen gegen die amerikanische Lösung, in der die Trennung zwischen föderalem und bundesstaatlichem Strafrecht nicht besonders eindeutig ist und zu vielseitigen Zuständigkeitskonflikten führt. ${ }^{52}$ Wenn wir uns dafür entscheiden, ein neues europäisches Strafrechtssystem einzuführen, wird dessen Einfluss auf das nationale Strafrechtssystem bei weitem geringer sein. ${ }^{53}$ Weiterhin würde es den Einwand entschärfen, dass nationale Prioritäten durch die Kompetenzabgabe an Europol und Eurojust vernachlässigt würden. ${ }^{54}$

\footnotetext{
Intersentia 2002, S.111. Die Normierung ist meist gemeinschaftsrechtlicher Herkunft; siehe J.A.E. Vervaele, Handen en tanden van het gemeenschapsrecht, Antrittsrede Utrecht 1994, S.36.

48 Siehe Dokumente der ersten Kammer des Parlaments, 2003-2004, 28350 (R1720) und 28351, B, S.2.

${ }^{49}$ A.H.J. Swart, Goede rechtsbedeling en internationale rechtshulp in strafzaken, Deventer 1983.

${ }^{50}$ Siehe auch Justizminister, Dokumente der ersten Kammer des Parlaments, 2003-2004, 28350 (R1720) und 28315, B, S.4).

${ }^{51}$ Die niederländische Regierung hat den europäischen Staatsanwalt ausdrücklich so charakterisiert, siehe Dokumente der ersten Kammer des Parlaments, 2003-2004, 28350 (R1720) und 28351, B, S.2.

521971 hat Enschedé das “American Model Penal Code” als Vorbild für ein europäischen Code abgelehnt, siehe Arnhem 1990.

${ }^{53}$ In verschiedenen Memorandi zum europäischen Strafrecht hat die Regierung die Prinzipien niederländischen Rechts aufgezählt, die undiskutierbar sind; Siehe das Memorandum zum Verhältnis zwischen Gemeinschaftsrecht und Strafrecht (Notitie betreffende de verhouding gemeenschapsrecht strafrecht) Dokumente der 2. Kammer des Parlaments, 1991-1992, 22300 VI, Nr.39; Memorandum zum Straf- und Strafprozessrecht im europäischen Kontext (Notitie Strafrecht en strafprocesrecht in Europees verband), Dokumente der 2. Kammer des Parlaments, 1998-1999, 26656, Nr.1.

${ }^{54}$ Der Justizminister hat richtigerweise in seiner Rede am 29. April 2003 darauf hingewiesen, veröffentlicht in Eurojust.
} 
In diesem Zusammenhang ist nicht alles rosig. Unweigerlich hat ein solches europäisches System auch seine Haken. Neue Organisationen werden gegründet und damit steigt der Datenfluss weiter an. Ein solches System muss auch psychologisch von den nationalen Behörden akzeptiert werde. ${ }^{55}$ Eine neue Institution darf nicht als Bedrohung oder Konkurrent angesehen und dadurch beim Informationsfluss benachteiligt werden. Die nicht gerade erfolgreiche Situation von Europol kann einen pessimistisch stimmen.

Was bedeutet dieses Modell für die Harmonisierung des Strafrechts und die gegenseitige Anerkennung?

Harmonisierung des nationalen Strafrechtssystems ist in dem System, das ich gewählt habe, nicht notwendig, da es einmal das europäische Strafrechtssystem für ganz bestimmte Straftaten gibt und ein nationales System für alle anderen. Die aktuelle Harmonisierung ist viel kritisiert worden und das mit Recht. Es beginnt mit der unklaren Prämisse, dass die doppelte Strafbarkeit der Kooperation in Strafsachen im Weg stünde. Die gewählte Methode ist kontraproduktiv. Der tatsächliche Einfluss der Harmonisierung ist bisher sehr geringfügig, wenn er nicht sogar völlig fehlt. ${ }^{56}$ Dies hängt in erster Linie mit der Gesetzgebung der Kompromisse zusammen. Rechtsinstrumente werden oft nur wage und nicht bindend verabschiedet. Jedem Mitgliedsstaat wird Raum gelassen, diese Instrumente selber zu interpretieren. Der zweite Gründ für die unterschiedliche Durchführung ist, dass die Umsetzung obligatorisch ist. Bei der Interpretation der Umsetzungspflichten auf einem „eher nationalen“ Wege kann ein Staat, der im Verhandlungsprozess eine Niederlage erlitten hat, am Ende doch noch seinen eigenen Weg gehen. ${ }^{57}$ Der dritte Grund besteht in der Nichtexistenz eines gemeinsamen Gerichtes. Selbst die momentane Rolle des Gerichtshofes bei der Auslegung von Rahmenentscheidungen muss noch festgelegt werden. Ein vierter Grund ist der unterschiedliche Sprachgebrauch. Das führt $\mathrm{zu}$ einer Situation, mit großen Unterschieden in der endgültigen Rechtsumsetzung in den verschiedenen Mitgliedsstaaten. Übermorgen wird die Europäische Union von 15 auf 25 Mitgliedsstaaten anwachsen, von 375 Millionen auf 450 Millionen Einwohnern. Diese Größe wird es noch schwieriger machen, einen gemeinsamen Nenner zu finden. Der Zwang zur Harmonisierung wird noch stärker werden.

Ausgehend von separaten Gerichtsbarkeiten - einer europäischen mit Hinblick auf spezifische europäische Verbrechen und verschiedene nationale für nationale Verbrechen - werden keine Bedingungen für eine doppelte Strafbarkeit benötigt. Dementsprechend ist ein Grund für die Harmonisierung materiellen Strafrechtes weggefallen. Nach meiner Einschätzung kann Harmonisierung einen noch formaleren Gesichtspunkt des Rechts erfüllen. Das erfordert eine Erklärung.

\footnotetext{
${ }^{55}$ Siehe A.H. Klip, Geïntegreerde bescherming van de financiële belangen van de EG; verschuiving van de staat-staat-benadering naar verticalisering van de procedure?, in: J.A.E. Vervaele (Hrgb.), Transnationale handhaving van de financiële belangen van de Europese Unie, Intersentia 1999, S.99111.

${ }^{56}$ Siehe beispielsweise T. VanderBeken, From Brussels with Love, Bespiegelingen over de invloed van de Europese Unie op het Belgisch strafrecht, Preadvies voor de Vereniging voor de vergelijkende studie van het recht van België en Nederland 2002. Siehe Weyembergh, S.336.

${ }^{57}$ Siehe Weyembergh, S. 342.
} 
Wenn die Mitgliedsstaaten gegenseitig akzeptieren, dass die Ausführung der Gerichtsbarkeit exklusiv ist, so ist das ein wichtiger Beitrag zur unbedingten gegenseitigen Anerkennung von Übergaben von Personen, Beweisstücken und anderen Beschlüssen. In einem System von unbedingter Anerkennung verschwinden die Schranken zwischen den Strafgesetzsystemen. ${ }^{58} \mathrm{Um}$ zu einem ganz freien Verkehr zu kommen, müssen verschieden Hindernisse beseitigt werden. Zum Beispiel unterscheiden sich die Verfahrensbedingungen für Festnahmen von Verdächtigen je nach Mitgliedsstaat. Das setzt die Staaten unter Druck, die striktere Bedingungen haben. Ähnliche Probleme können bei anderen Zwangsmitteln oder der Beweissammlung auftauchen. Die Annäherung der Bedingungen, die erfüllt sein müssen bevor eine Durchsuchung und Verhaftung stattfinden dürfen, wird die Einwände gegen Anerkennung fremder Rechtsordnungen verschwinden lassen.

Solche Ergebnisse zeigen wie nötig ein Konzept für ein Prinzip der gegenseitigen Anerkennung ist. Was genau muss anerkannt werden? Dass genau die gleichen materiellen Bedingungen erfüllt sein müssen, bevor ein Zwangsmittel angewendet werden darf? Dass eine einzelne Behörde diese festgelegt hat? Dass der ersuchte Staat die Prozessregeln zur Beweissammlung anwendet, die der ersuchende Staat anwendet? Und warum sollte der obligatorische Vollzug einer ausländischen Anfrage störend in die staatseigene Justizverwaltung eingreifen? Kurzum: der Begriff muss mit einem Konzept gefüllt werden.

In der heutigen Situation bestimmt der Staat, der die Initiative ergreift, dass ein anderer Staat etwas anerkennen soll. Warum sollte in einem System der gegenseitigen Anerkennung ein Staat, der eine Tat strafrechtlich verfolgt, den Vorrang erhalten über einen anderen Staat, der sich dafür entschieden hat, keine Konsequenzen an eine bestimmte Tat zu knüpfen. Mit einer Einschränkung der Gerichtsbarkeit muss eine solche Frage nicht länger diskutiert werden.

Wie würde ein europäisches Strafrecht aussehen?

Im Vorfeld hab ich über die Abgrenzung zwischen nationalem und europäischem Strafrecht gesprochen, aber ich habe noch nichts zu der Ausgestaltung des europäischen Strafrechtes gesagt. Wie könnte es aussehen?

Grundsätzlich gibt es zwei verschiedene Methoden, wie einem europäischen Strafrecht und Strafprozessrecht Gestalt und Gehalt gegeben werden können. Schon in diesem Stadium müssen wir auch an das Strafprozessrecht denken, da es doch immerhin um die Erschaffung eines neuen Systems geht. Die erste Methode leitet das System von der Arbeit ab, die schon von der Europäischen Union geleistet wurde. Die zweite ist es, von gemeinsamen strafrechtlichen Traditionen abzuleiten. Beide Übungen werden alles andere als einfach sein. Das ist nicht erstaunlich. Es wäre erst das dritte Mal seit christlicher Zeitrechnung, dass ein Versuch unternommen wird, ein gemeinschaftliches Strafrechtssystem zu erschaffen. Nach dem römisch-kanonischen Strafrecht und Napoleons Code Pénal haben sich die Umstände nicht erheblich verändert.

${ }^{58}$ A.H.J. Swart, Een ware Europese rechtsruimte, Antrittsrede Amsterdam, Deventer 2001, S.24. 
Was bedeutet es, den gemeinsamen Nenner aus den Rechtsinstrumenten abzuleiten, die bereits von der Union akzeptiert wurden? Der materielle Teil des Strafrechtes wird durch die Kausalhaftung ausgezeichnet. ${ }^{59}$ Das tatsächliche Verhalten des Täters ist nicht wirklich von Wichtigkeit. Es geht um den Posten oder die Verantwortung, die dieser übernimmt (Sorgfaltspflichtsverletzung).Vielleicht wird das verursacht, in dem man auf das Recht zur Verwirklichung des Binnenmarktes aufbaut. Die Instrumente der Europäischen Union enthalten Elemente, die zum allgemeinen Teil des Strafrechts in jedem der Rechtssysteme gehören. Da es bisher auf Unionsebene zu diesem Thema noch nicht systematisch nachgedacht wurde, gibt es viele lose Ideen. Sie sagen uns etwas darüber, wen die Union als Verbrecher ansieht, wie sie Täterschaft und Teilnahme an einem Verbrechen definiert und auf welche Weise ein Delikt aufgebaut ist. Fast ausnahmslos handelt es sich um sehr generelle Definitionen von Mittäterschaft an einer Straftat.

Die geforderte Absicht manifestiert sich in einem Verhalten (Artikel 1 Abs. 4 der EG Betrugskonvention). Weiterhin muss man Haftung von juristischen Personen und ihren formellen wie tatsächlichen Führungskräften in Betracht ziehen (Art. 6 des Rahmenbeschlüsses zur Bekämpfung der sexuellen Ausbeutung von Kindern und der Kinderpornographie). Wenn strafrechtliche Haftung schon auf der Kompetenz gegründet sein kann, eine juristische Person zu repräsentieren, Entscheidungen in ihrem Namen zu treffen oder sie zu beaufsichtigen, so ist die Bedeutung von Schuldausschließungsgründen und Rechtfertigigungsgründen stark gemindert.

In der Bewertung der Zurechnung des Täters liegt bereits ein Urteil über die eventuelle Anwendbarkeit von Strafausschließungsgründen. Abgesehen davon können - besonders in den Präambeln - einige Elemente der Strafrechtspolitik entdeckt werden, die das materielle Strafrecht beeinflussen könnten. Die EU-Norm strebt nach der endgültigen Ausschaltung strafbaren Verhaltens (oder gar der Straftäter) und nach Rückfallprävention.

Diese Methode bringt nicht viel Einblick in die Entwicklung des Strafprozessrechts. Das Recht der dritten Säule beinhaltet relativ wenig strafverfahrensrechtliche Vorschriften. Das ist deswegen logisch, da Strafverfolgung bislang eine nationale Angelegenheit ist. Die Betonung liegt besonders auf der Harmonisierung (und der Erleichterung) der Zusammenarbeit in Strafsachen.

Um mit Hilfe der ersten Methode festzustellen, was europäisches Strafrecht sein könnte, habe ich einige Dinge vom bestehenden EU-Recht abgeleitet. Bei der zweiten Methode wird europäisches Strafrecht von den gemeinsamen Strafrechtstraditionen der Mitgliedsstaaten abgeleitet. ${ }^{60}$ Auch hier sind der allgemeine Teil des Strafrechtes (Gesetzmäßigkeit, Täterschaft und Teilnahme, Vorsatz und Schuld, etc.) und das

\footnotetext{
${ }^{59}$ Eine Entwicklung, die sich besonders in der Rechtssprechung der internationalen Strafgerichtshöfe zeigt.

${ }^{60}$ Eine derartige Ableitungsmethode ist vergleichbar mit dem, was Harmonisierung tut. Bildlich könnte man sagen, dass das Ableiten der Prinzipien verschiedener Systeme eine Art Trichtermethode ist. In der klassischen Harmonisierung stellt man den Trichter dagegen auf den Kopf.
} 
Strafprozessrecht wichtiger als einzelne Straftatbestände. ${ }^{61}$ Artikel 6 des EUVertrages bietet eine rechtliche Grundlage, um dies von den kulturellen Traditionen der Mitgliedsstaaten herzuleiten. Daneben haben wir die Rechtsprechung des Europäischen Gerichtshofes zu den Verfassungs- und anderen nationalen Traditionen der einzelnen Staaten. Auch sind grundlegende Prinzipien des Gemeinschaftsrechts entwickelt worden. ${ }^{62}$

Was bringt eine solche erste Bestandsaufnahme? Zuallererst die Grundrechte, die auch im Strafrecht von Bedeutung sind. Dank der vereinheitlichenden Rolle der EMRK besteht ein einigermaßen übereinstimmendes Bild. Wenn wir die gleiche Methode für andere Aspekte des Strafrechts anwenden, entsteht ein gemischteres Bild. $^{63}$

Es gibt einige Unterschiede in den Rechtskulturen, was Suche nach Parallelen erschwert. Mehr oder weniger gleiche Regelungen können sehr unterschiedlich interpretiert werden. Das Legalitätsprinzip zum Beispiel, nach dem niemand für eine Tat haftbar gemacht werden kann, gegen die es vorher noch kein Gesetz gab, wird zu verschiedenartigen Interpretationen zu den Bedingungen an die Rechtsklarheit (lex certa) führen. Die Rechtskultur sagt uns auch etwas über die Wahrnehmung des Rechts eines anderen Systems. Beispielsweise kritisieren viele Länder, die das Opportunitätsprinzip bei der Verfolgung von Verdächtigen anwenden, solche Länder, die sich nach dem Legalitätsprinzip richten, da dies in ihren Augen zu einer Sturheit und einem extremen Gebrauch des Strafrechts führt. Unterdessen wird umgekehrt dem Opportunitätsprinzip Willkür vorgeworfen. Tatsächlich haben beide die scharfen Kanten ihrer Systeme genommen und haben so die Kritik überwunden. Die Diskussion, die in den Niederlanden über Mindeststrafen stattfand, ist ein anderes Beispiel für die Gefahren der Interpretation fremden Rechts. Diese wurde von einem niederländischen Standpunkt aus geführt, der von der strikten Ausführung der verhängten Strafen ausgeht (mindestens 2/3 werden abgeleistet). Auf der anderen Seite haben Länder, in denen es Mindeststrafen gibt, lauter Ausnahmen eingeführt. Frankreich hat sie gänzlich aus seinem Gesetzbuch genommen, da Richter schon für wenig schwere Tathandlungen verurteilten, weil sie sonst in ihren Augen gezwungen wären, zu harte Urteile zu fällen. ${ }^{64}$

Dies sind nur einige Beispiele, aber sie zeigen, dass die Suche nach dem größten gemeinsamen Nenner zu einem vollkommen neuen System führen wird, in dem keines der nationalen Systeme sich vollends wieder finden wird. Was die Entwicklung eines neuen Strafprozessrechts angeht, so kann die Erfahrung von internationalen Strafgerichtshöfen von Hilfe sein.

\footnotetext{
${ }^{61}$ Siehe beispielhaft W. Bottke, Die Struktur von Täterschaft bei aktiver Begehung und Unterlassung als Baustein eines gemeineuropäischen Strafrechtssystems, in: Bausteine des europäischen Strafrechts, Coimbra-Symposium für Claus Roxin, 1995, S.235-246.

${ }^{62}$ Einige Beispiele: EuGH, 5. März 1980, Ferwerda, C-256/78, Jur.1980, 623: EuGH, 13. November 1990, Fedesa, C-331/88, Jur.I-4057; EuGH, 4. Oktober 1991, SPUC, C-159/90, Jur.1991, 4658; EuGH, 14. Mai 1974, Nold, C-4/73, Jur.1974, 491; EuGH, 13. Dezember 1979, Hauer, 44/79, Jur.1979, 3727; EuGH, 21. September 1989, Hoechst, C-46/87 und C-227/88, Jur.1989, 2859.

${ }^{63}$ Natürlich gibt es mehr Strafprozessrecht als in der EMRK, siehe M. Delmas-Marty, Procédures pénales d'Europe, Paris 1995.

${ }^{64}$ A.M. van Kalmthout en P.J.P. Tak, Ups en downs van de minimumstraf, Een verkennende studie naar het voorkomen van minimumstraffen in Frankrijk, België, Duitsland, Engeland en Wales, Nijmegen 2003.
} 
Es gibt noch einen anderer Aspekt des Aufbaus eines europäischen Strafrechtssystems, der diskutiert werden muss, und das ist die Sprache. Bei einem gemeinsamen europäischen Strafrecht scheint es mir unvermeidbar, eine einzige rechtliche Sprache zuhaben. Weil Sprache auch Rechtssprache ist, führt der Gebrauch unterschiedlicher Sprachen zu unterschiedlichen Interpretationen. Benutzt man Begriffe wie opzet (Vorsatz) oder nalaten (Unterlassung), hat das automatisch eine niederländische - wenn Sie wollen eine belgische - Erklärung zur Folge. Jeder, der sich etwas mit Rechtsvergleich auskennt, weiß, dass die deutschen Begriffe Vorsatz und Unterlassung zwei völlig unterschiedliche Konzepte sind, die in ihren eigenen Zusammenhang passen. Sollte die Umsetzung europäisch, also gemeinschaftlich werden, bleibt keine Platz mehr für die Unterschiede, die sich aus den verschiedenen Sprachen ergeben. In dem Bestreben nach Integration und Harmonisierung baut sich die Union große Hindernisse dadurch auf, dass sie immer noch alle europäischen Sprachen für gleichermaßen verbindlich hält. ${ }^{65}$ Ab übermorgen werden es zwanzig sein!

Mit der Wahl für eine könnte eine europäische Rechtssprache entstehen. Es liegt auf der Hand, dass die Wahl wahrscheinlich auf Englisch fallen würde. Das könnte problematisch sein, denn es kann unterstellt werden, dass die größeren Gemeinsamkeiten der Strafrechtssysteme der Mitgliedsstaaten in einem zivilrechtlichen System liegt. Erfahrungen in verschiedenen internationalen Strafgerichten zeigen, dass ein völlig neues System entsteht, und dass es seine eigene Balance findet, auch wenn die Regelungen aus ganz verschiedenen Systemen kommen. Deswegen sollte es möglich sein, dass die europäische Rechtssprache Englisch sich von ihrem nationalen Kontext befreit. Solange das noch nicht passiert ist, und solange die Umsetzung national geschieht, bleibt das Niederländische die Rechts- und auch die Wissenschaftssprache. Das erfordert, dass in der Wissenschaft auf Niederländisch geschrieben und gelehrt wird, so wie auch diese Rede ursprünglich auf Niederländisch gehalten wurde.

\section{Europäisierung auf der Ebene des Rechtsschutzes}

In meinem Expose über die Definition der Gerichtsbarkeit in den einzelnen Staaten und die Schaffung einer europäischen Kompetenz im Strafrecht habe ich kaum über den damit zusammenhängenden Schutz der Grundrechte gesprochen. Die Europäische Union vergisst oft, dass transnationale Rechtsumsetzung oft selbst neue Fragen des Rechtsschutzes aufwirft. Zuallererst betrifft das Fälle, in denen Länder in strafrechtlichen Angelegenheiten zusammenarbeiten. Grundrechtsschutz wird momentan eher national und isoliert behandelt. Die Anpassung dieses Schutzes zu einer grenzübergreifenden Kooperation ist dringend notwendig. ${ }^{66}$ Orie beschrieb dies schon 1983 in seinem berühmten Aufsatz über den „Systembruch in der

\footnotetext{
${ }^{65}$ Siehe unter anderem W.C. van Binsbergen, Integratie, Een revolutie? Antrittsrede Utrecht 1963, S.12.

${ }^{66}$ Siehe für die Position des Beklagten in einem transnationalen Fall: T. Spronken, A place of greater safety, Bespiegelingen over een Europees Statuut voor de strafrechtsadvocaat, Antrittsrede Maastricht 2003.
} 
internationalen Rechtshilfe“. ${ }^{67}$ In den meisten solcher Fälle fehlt es den Verdächtigen und anderen beteiligten Personen sowohl an Rechtsmitteln im ersuchenden Staat als auch im ersuchten Staat. Im ersuchenden Staat deswegen, da man sich schlecht über illegale Handlungen beschweren kann, die in einem fremden Land unter der Verantwortung von fremden Staatsdienern stattgefunden haben. Und im ersuchten Staat sind keine Mittel möglich, da die Straftat dort nicht verfolgt wird, und da internationale Kooperation in Strafangelegenheiten oft zu einem Zeitpunkt stattfindet, in der der Verdächtige sich gar nicht im Klaren darüber ist, dass er Objekt von Ermittlungen ist. Die Weigerung anzuerkennen, dass es transnationale Umsetzung gibt, unterstützt diesen Systembruch. In dieser Hinsicht ist es nicht übertrieben zu sagen, dass das Festhalten an einer strikt nationalen Strafrechtsvollstreckung (und damit das Bekämpfen einer Europäisierung des Strafrechtssystems) für den Rechtsschutz von Verdächtigen und anderen beteiligten Personen nachteilig ist. Die Erhaltung zweier Rechtssicherungssysteme beschränkt den eigentlichen Rechtsschutz. Insofern ist ein plus ein null.

Die gegenseitige Anerkennung der Staaten von ihren jeweiligen Handlungen muss alle Aspekte der Hilfestellung beinhalten. Dabei können im Hinblick auf die Verantwortung der Mitgliedsstaaten bei Handlungen auf der Grundlage der EMRK keine Ausnahmen gemacht werden. Ein Staat, der sich der Leistung eines anderen Mitgliedsstaates bedient, muss im Falle von Menschenrechtsverletzungen die Verantwortung übernehmen. Für kooperierende Staaten bedeutet das, dass sie nicht nur die Erleichterungen, sondern auch die Lasten der ausländischen Hilfe tragen müssen. Auf diese Weise kann von der relativen Willkür der momentanen Situation Abstand genommen werden. Wenn der Kläger rechtzeitig herausfindet, welche Staaten in die Untersuchungen gegen ihn involviert sind, so muss er raten, gegen welchen von ihnen er seine Beschwerde richten muss - gegen den ersuchten oder den ersuchenden Staat. Der Europäische Menschenrechtshof und die Europäische Menschenrechtskommission haben eine gemeinschaftliche Verantwortung immer abgelehnt. $^{68}$

Beispielhaft ist der Sari Fall. Der EMRH erklärte eine Beschwerde für unzulässig, die eine Verletzung der angemessenen Frist des rechtlichen Gehörs gegen Dänemark und die Türkei zum Inhalt hatte. Zwar dauerte der gesamte Vorgang der Übertragung der Strafverfolgung und die folgende Gerichtsverhandlung mehr als zehn Jahre, aber jeder der Staaten übernahm nur die Hälfte der Verantwortung dafür, und das war dem Gerichtshof zufolge individuell gesehen nicht einmal unverschämt. ${ }^{69}$ Meiner Meinung nach sollte es nicht in der Zuständigkeit des Beschwerdeführers liegen, die genaue Verantwortung der Staaten festzulegen, die in der Menschenrechtsverletzung zusammenarbeiten. Denn es geht ja darum, dass das gemeinsame Handeln der betroffenen Staaten gegen ihn nicht rechtens war. Auf der Grundlage des Artikels 1 (i.V.m. der Präambel) der Europäischen Menschenrechtskonvention ist es eine gemeinsame Pflicht aller Vertragsparteien, eine Verletzung dieser zu vermeiden. Ich halte das für eine Art persönliche Haftung. Bevor ein Strafverfahren als Ganzes

\footnotetext{
${ }^{67}$ A.M.M. Orie, De verdachte tussen wal en schip of de systeem-breuk in de kleine rechtshulp, in: Bij deze stand van zaken, bundel opstellen aangeboden aan A.L. Melai, Arnhem 1983, S.351-361.

${ }^{68}$ Siehe A.H. Klip, Die EMRK und die internationale Zusammenarbeit in Strafsachen, Universität Halle (im Druck) und ebenso The Decrease of Protection under Human Rights Treaties in International Criminal Law, 68 Revue Internationale de Droit Pénal 1997, S.291-310.

${ }^{69}$ EuGMR, 8. November 2001, Sari gegen Dänemark und die Türkei, 21889/93, par.91 und 99.
} 
beurteilt wird, sollte ein Bürger in jedem beteiligten Staat rechtliche Mittel einsetzen können.

In Zukunft muss die Umsetzung des Strafrechts durch die Europäische Union von deren Beitritt zur Europäischen Menschenrechtskonvention bedingt sein. Das bietet einerseits einen rechtlichen Schutz, der mit den Traditionen der einzelnen Mitgliedsstaaten übereinstimmt. Außerdem gewährt es weit mehr Schutz als die seltsame und relativ begrenzte Auswahl an Menschenrechten im Bereich des Strafrechts, die in der Menschenrechtscharta der EU und dem Verfassungsentwurf niedergeschrieben sind. ${ }^{70}$

\section{Abschluss}

Ich komme zum Ende.

Eine Menge Wasser wird noch die Maas hinab fließen bis die Europäische Union mit der systematischern Gestaltung eines Strafrechtssystems beginnen wird. Das hängt mit den verschiedenartigsten institutionellen Aspekten der Verwirklichung eines gemeinsamen Strafrechtssystems und mit der Frage der politischen Machbarkeit zusammen. Das so genannte Debakel von Dezember 2003, als die europäische Verfassung noch nicht zustande gekommen ist, kann für die Rechtsentwicklung nur günstig sein. Der Unionsgesetzgeber kann die Zeit nutzen, um die Qualität der Rechtsmittel zu verbessern. Dabei ist wichtig, dass das neue System die Ansprüche verschiedener Menschenrechtsabkommen erfüllt und dass es ein Strafrecht erschafft, das umsetzbar ist. Das kann zu einem Weniger an Strafrecht führen, aber nur ein realisierbares Strafrecht kann eine wertvolle Rolle in der europäischen und niederländischen Gesellschaft spielen.

\footnotetext{
${ }^{70}$ Siehe auch Art. II-47 - 50 des europäischen Verfassungsentwurfes und Art. 47-50 der Menschenrechtscharta der EU.
} 